\title{
Progesterone in Women with Bleeding in Early Pregnancy: Absence of Evidence, Not Evidence of Absence
}

\author{
Quentin F. Gronau and Eric-Jan Wagenmakers \\ University of Amsterdam
}

A recent trial assessed the effectiveness of progesterone in preventing miscarriages. ${ }^{1}$ The number of live births was $74.7 \%(1513 / 2025)$ in the progesterone group and $72.5 \%$ $(1459 / 2013)$ in the placebo group $(p=.08)$. The authors concluded: "The incidence of adverse events did not differ significantly between the groups."

This conclusion leaves unaddressed the degree to which the data undercut or support the progesterone hypothesis. To quantify such evidence we conducted Bayesian logistic regression. ${ }^{2,3}$ Under the no-effect model $\mathrm{H}_{0}$, the log odds ratio equals $\psi=0$, whereas under the positive-effect model $\mathrm{H}_{+}, \psi$ is assigned a positive-only normal prior $\mathrm{N}_{+}(\mu, \sigma)$. A default analysis (i.e., $\mu=0, \sigma=1$ ) reveals only weak evidence for $\mathrm{H}_{0}{ }^{4}$ Figure 1 shows the evidence is weak for all combinations of $\mu$ in $[0,0.30]$ and $\sigma$ in $[0.25,1]$. R code for reproducing the analyses is available from https://osf.io/tu4sv/.

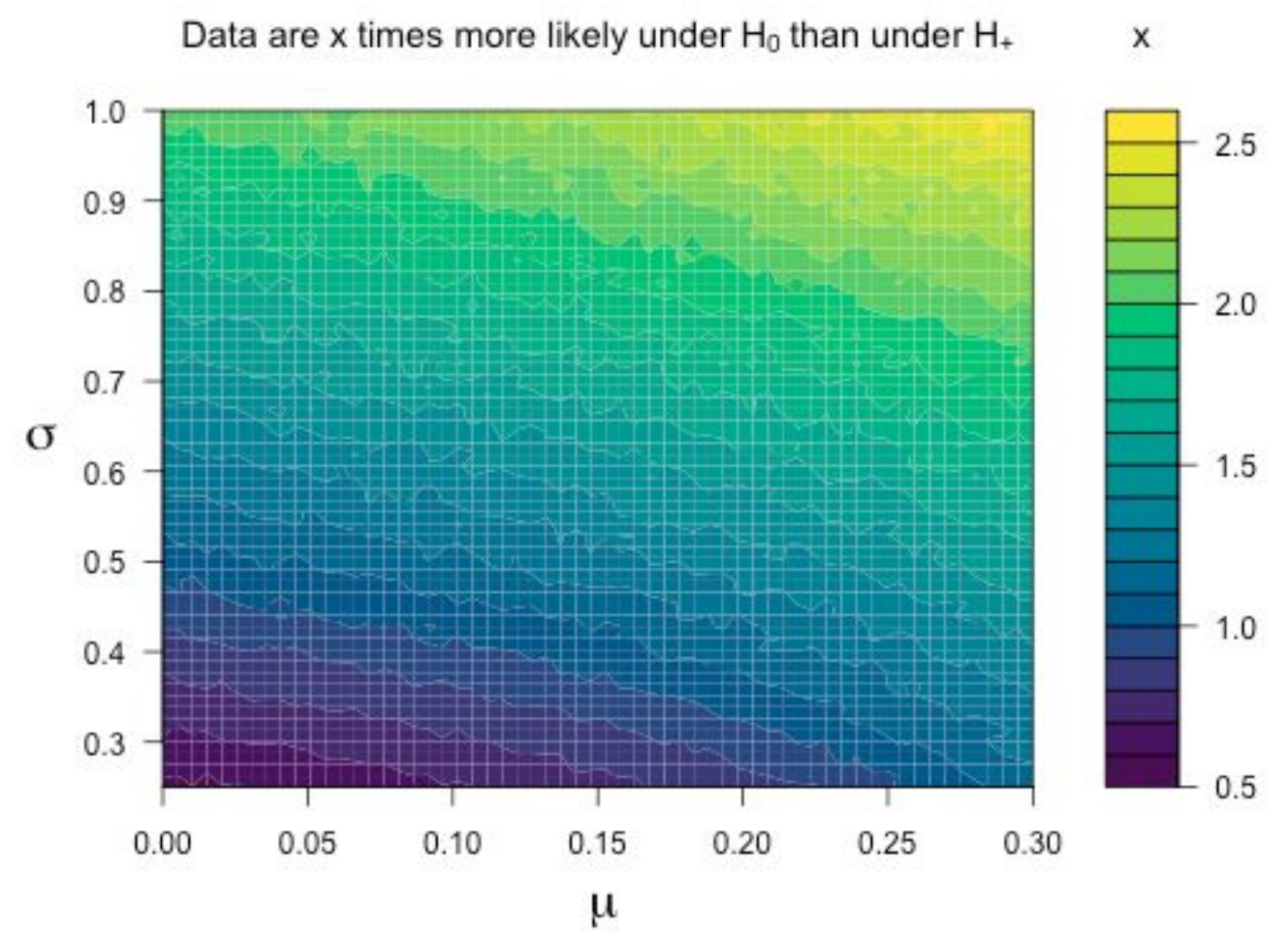

Figure 1. Across different priors, the evidence for no-effect $H_{0}$ over positive-effect $H_{+}$is weak.

In sum, these data neither undercut nor support the progesterone hypothesis in compelling fashion. 


\section{References}

1. Coomarasamy A, Devall AJ, Cheed V, et al.. A randomized trial of progesterone in women with bleeding in early pregnancy. New England Journal of Medicine 2019;380:1815-24.

2. Kass RE, Vaidyanathan SK. Approximate Bayes factors and orthogonal parameters, with application to testing equality of two binomial proportions. Journal of the Royal Statistical Society: Series B (Methodological) 1992;54:129-44.

3. Gronau QF, Raj K. N. A., Wagenmakers EJ. (2019). Informed Bayesian inference for the $A / B$ test. Manuscript submitted for publication and available on arXiv:

http://arxiv.org/abs/1905.02068

4. Jeffreys, H. Theory of Probability. 1st ed. Oxford University Press, Oxford, UK, 1939.

Conflict of Interest Statement: There are no conflicts of interest to report.

\section{Authors}

Quentin F. Gronau

Department of Psychological Methods

University of Amsterdam

Nieuwe Achtergracht 129 B

1018 WT Amsterdam, The Netherlands

E-mail: Quentin.F.Gronau@gmail.com

Eric-Jan Wagenmakers

Department of Psychological Methods

University of Amsterdam

Nieuwe Achtergracht 129 B

1018 WT Amsterdam, The Netherlands

E-mail: EJ.Wagenmakers@gmail.com 